\title{
Clinical efficacy of tocilizumab in patients with active rheumatoid arthritis in real clinical practice
}

\author{
Yasuhiko Hirabayashi · Tomonori Ishii · Hideo Harigae
}

Received: 31 May 2009 / Accepted: 7 August 2009 / Published online: 22 August 2009

(c) The Author(s) 2009. This article is published with open access at Springerlink.com

\begin{abstract}
The previous clinical studies have demonstrated tocilizumab monotherapy to be highly effective in rheumatoid arthritis (RA). The objectives of the present article are to report the efficacy and safety of tocilizumab in patients with active RA in real clinical practice. In total, 61 patients with RA were treated with tocilizumab. Any comorbidities they had, especially infections, were treated thoroughly before they were given the drug. We provided guidance on infection control and prevention. Mean age of the patients was $60.9 \pm 12.4$ years, and their mean disease duration $10.9 \pm 9.2$ years. The patients remained on steroids, methotrexate, and tacrolimus as before, but were taken off any other drugs they had been using prior to the treatment. Mean of the 28-joint disease activity score using erythrocyte sedimentation rate was $4.75 \pm 1.15$ initially and fell to $2.21 \pm 0.97$ after two doses $(n=50)$. After four doses, the remission rate was $83.8 \%$ (31/37). All patients responded well to the therapy and there was no decrease in the efficacy of tocilizumab during the treatment. Even in the real clinical setting, treatment with tocilizumab can rapidly induce remission in RA in a high proportion of patients and is generally safe and well tolerated. Tocilizumab would seem to be promising as a first-line choice for the treatment of RA.
\end{abstract}

\footnotetext{
Y. Hirabayashi ( $\square)$

Department of Rheumatology,

Hikarigaoka Spellman Hospital, 6-7-1 Higashisendai,

Miyagino-ku, Sendai 983-0833, Japan

e-mail: y_hirabayashi@ doctor.nifty.jp

Y. Hirabayashi $\cdot$ T. Ishii $\cdot$ H. Harigae

Department of Rheumatology and Hematology,

Tohoku University Graduate School of Medicine,

1-1 Seiryo-cho, Aoba-ku, Sendai 980-8574, Japan
}

Keywords Rheumatoid arthritis · Tocilizumab · Interleukin-6 · DAS28ESR

\section{Introduction}

The characteristic pathophysiology in rheumatoid arthritis (RA) is the destruction of bone and cartilage due to persistent synovitis of unknown etiology. Large quantities of inflammatory cytokines such as tumor necrosis factor (TNF), interleukin-1 (IL-1), and interleukin-6 (IL-6) are produced in inflamed synovial membranes, and these are deeply involved in the spread and persistence of the inflammation [1]. The basic concept behind anti-cytokine therapies is to trap these inflammatory cytokines using biological agents such as antibodies or receptors and so block their activity.

TNF inhibitors are a recent development in this field and have been demonstrated to be useful in the treatment of RA [2]. The progression of bone destruction can be halted by using them in combination with methotrexate (MTX) [3]. However, they may also increase susceptibility to serious infections such as tuberculosis. Even when MTX is used concomitantly, there are also instances of primary nonresponders, mid-treatment secondary non-responders (escape cases), and inadequate responders, indicating that TNF is of little or no relevance to the condition in some patients. Drugs able to overcome the limitations of antiTNF therapy have thus been sought.

Tocilizumab (TCZ) is a humanized monoclonal antibody against IL-6 receptors and inhibits binding between IL-6 and IL- 6 receptors $[4,5]$. It has been shown to be useful against RA in the clinical trials conducted to date. In the SATORI study, it gave significantly higher improvement rates than MTX [6], in the OPTION study, clinical efficacy 
was higher with MTX + TCZ than with MTX alone [7], in the SAMURAI study, it was effective as monotherapy for controlling the progression of bone and joint destruction [8], and in the STREAM study, it provided high levels of efficacy and continuity of response as long-term monotherapy [9]. Mean 28-joint disease activity score using erythrocyte sedimentation rate (DAS28ESR) at baseline had been elevated in the clinical trials ( 6.1 in the SATORI and 6.5 in the SAMURAI study), and the remission rate achieved was about $40 \%$ at 24 weeks in the SATORI study and $59 \%$ at 52 weeks in the SAMURAI study.

TCZ was approved for marketing in Japan for use in patients with RA on 16 April 2008. This was the first time that the drug had been approved anywhere so there are still almost no reports of treatment outcomes in real clinical practice. We evaluated our clinical experience with TCZ for therapy of RA. Here, we report the clinical efficacy and safety of TCZ in the treatment of patients with active RA in real-world settings.

\section{Patients and methods}

Patients

The subjects of this analysis were patients who met the 1987 revised criteria for the classification of RA from the American College of Rheumatology (ACR) and who had active disease with the DAS28ESR of 2.6 or over. Patients were included in the analysis if they started treatment with TCZ for the first time in the 11 months between 16 April 2008 (the time of insurance approval in Japan) and 15 March 2009 and they received TCZ at least twice during this time. Their demographics, baseline characteristics, and drug use prior to the treatment are shown in Table 1. The criteria for exclusion from the analysis set were as follows: (i) autoimmune disease comorbidities except Sjögren's syndrome and Hashimoto's disease, (ii) functional class IV based on the Steinbrocker criteria, (iii) presence of active infection, (iv) pregnancy, (v) drug poisoning, including alcohol, (vi) lymphocyte count $\leq 500$ cells $/ \mu$ l, and (vii) positive serum $\beta$-D-glucan.

\section{Screening}

To reduce adverse events, any comorbidity was treated or controlled as well as possible before giving TCZ. All patients had a thoracic CT scan and were tested for the tuberculin reaction, anti-streptolysin O (ASO), anti-streptokinase (ASK), treponema pallidum haemagglutination (TPHA), hepatitis B surface antigen, anti-hepatitis $\mathrm{C}$ virus antibody, and $\beta$-D-glucan to screen for infections. If tooth plaque or caries were present, we arranged for assessment
Table 1 Patient demographics, clinical characteristics, and previous medications at baseline

\begin{tabular}{|c|c|}
\hline \multicolumn{2}{|l|}{ Demographics } \\
\hline Age (years) & $60.9 \pm 12.4$ \\
\hline Male:Female & $12: 49$ \\
\hline \multicolumn{2}{|l|}{ Clinical characteristics } \\
\hline RA duration (years) & $10.9 \pm 9.2$ \\
\hline Steinbrocker Class (I, II, III, IV) & $25,25,11,0$ \\
\hline Steinbrocker Stage (I, II, III, IV) & $11,9,5,36$ \\
\hline Tender joint count $(0-28)$ & $5.3 \pm 4.9$ \\
\hline Swollen joint count $(0-28)$ & $4.0 \pm 3.5$ \\
\hline $\operatorname{ESR}(\mathrm{mm} / \mathrm{h})$ & $39.8 \pm 25.6$ \\
\hline $\mathrm{CRP}(\mathrm{mg} / \mathrm{l})$ & $17 \pm 17$ \\
\hline DAS28 (4/ESR) & $4.75 \pm 1.15$ \\
\hline \multicolumn{2}{|l|}{ Previous medications } \\
\hline Prednisolone ( $n=, \mathrm{mg} /$ day) & $45,3.95 \pm 3.12$ \\
\hline Anti-TNF $(n=)$ & 6 \\
\hline $\operatorname{MTX}(n=)$ & 21 \\
\hline Tacrolimus $(n=)$ & 5 \\
\hline Other DMARDs $(n=)$ & 28 \\
\hline \multicolumn{2}{|c|}{ The values shown are mean \pm SD unless otherwise indicated } \\
\hline \multicolumn{2}{|c|}{ CRP only calculated from data for 60 patients } \\
\hline \multicolumn{2}{|c|}{$\begin{array}{l}\text { 'Previous medications' denotes drugs used in the } 3 \text { months before } \\
\text { administration of TCZ }\end{array}$} \\
\hline \multicolumn{2}{|c|}{$\begin{array}{l}\text { 'Other DMARDs' were sodium aurothiomalate, bucillamine, sala- } \\
\text { zosulfapyridine, and mizoribine. These were discontinued on initiating } \\
\text { TCZ }\end{array}$} \\
\hline
\end{tabular}

and treatment by a dentist, including for the presence of apical periodontitis. If chronic rhinorrhea or nasal blockage was seen, the patient was assessed and treated by an otolaryngologist. Patients were asked whether they had hemorrhoids.

Handling of previous medications

Among the various therapies being used by the patients in the three months before they received TCZ, infliximab were discontinued at least one-month before and etanercept at least 4 days before the new treatment commenced. Salazosulfapyridine, bucillamine, sodium aurothiomalate, and mizoribine were discontinued upon initiating TCZ. The patients stayed on methotrexate, tacrolimus, and steroids at the same dose levels as before at least until dose 3 of TCZ. There were no users of adalimumab, auranofin, D-penicillamine, hydroxychloroquine, minocycline, or lobenzarit disodium, nor had any patient undergone granulocytapheresis therapy.

\section{Administration of TCZ}

The method of administering TCZ that has received insurance approval in Japan is a continuous infusion of a dose of 
$8 \mathrm{mg} / \mathrm{kg}$ over a period of at least $1 \mathrm{~h}$ once every 4 weeks. No conditions have been set for finishing treatment. The interval between infusions was shortened to 3 weeks between the first and second infusions only, and an interval of $4 \pm 1$ weeks was used thereafter. Treatment was continued unless there were adverse events requiring discontinuation of treatment or the patient requested that treatment be stopped.

\section{Patient education}

We provided guidance on infection control and prevention. We also gave lifestyle advice to try and achieve standard body mass index (BMI).

\section{Statistical analysis}

Statistical significance was determined by the two-tailed Student's $t$-test with $P<0.05$ taken to indicate significance.

\section{Results}

\section{Clinical efficacy}

Mean DAS28ESR at the start of TCZ treatment in all 61 patients was $4.75 \pm 1.15$. After two doses, mean DAS28ESR had fallen below the remission threshold $(<2.6)$ to $2.21 \pm 0.97$ and after six doses had further improved to $1.63 \pm 0.71$ (Fig. 1a). The clinical response was evaluated using the European League Against Rheumatism (EULAR) response criteria. Thirty-one out of 37 patients $(83.8 \%)$, who had received four doses, had achieved remission with treatment response of good in $97.3 \%$, moderate in $2.7 \%$, and no cases of no response (Fig. 1b). The no response seen beyond five doses was due to a rise in DAS28ESR associated with a temporary deterioration in symptoms due to factors other than RA and did not represent secondary non-response. To date, no patient has discontinued the treatment due to lack of response.

The doses of concomitant drugs after starting treatment with TCZ were examined in 18 patients who received TCZ at least six times (for approximately 6 months) in the observation period. Of these 18 patients, 16 used steroids, 5 used MTX, and none used tacrolimus. In all five patients who used MTX, tapering was completed after remission, but RA did not flare up. The steroid dose was decreased slowly to avoid steroid withdrawal syndrome. In the 16 patients who used steroids, the mean steroid dose was $5.4 \mathrm{mg}$ /day (prednisolone equivalent) at the start of treatment with TCZ and $4.1 \mathrm{mg} /$ day at the end of the observation period. In all five patients who were using tacrolimus at the start of treatment with TCZ, it was possible to reduce the dose of tacrolimus
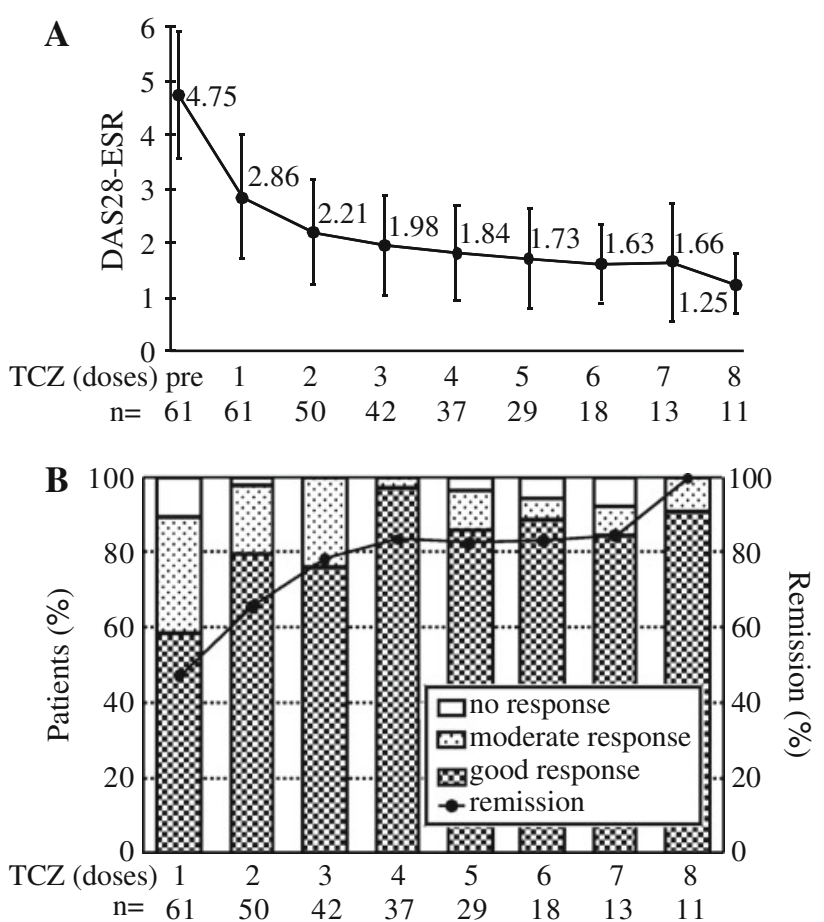

Fig. 1 a Change in DAS28ESR in all patients. Mean values shown. Bars indicate SD. " $n$ " indicates the number of patients. Since each patient was treated with TCZ starting on the different dates, " $n$ " in each "doses", indicated in abscissa, decreases dependent on the date of the first dose. $\mathbf{b}$ Change in response and remission rate in all patients

or to finish treatment with tacrolimus in the observation period, even though the patients had received TCZ not more than five times.

Next, we investigated differences in the therapeutic efficacy of TCZ depending on their baseline level of disease activity. We classified the patients based on DAS28ESR into a high-activity group at $>5.1 \quad(n=23)$, a moderateactivity group at 3.2-5.1 $(n=32)$, and a low-activity group at $<3.2(n=6)$. Although mean DAS28ESR had been $5.87 \pm 0.75$ in the high-activity group before treatment with TCZ, this improved rapidly and went below the remission threshold to $2.27 \pm 0.98 \quad(n=16)$ after three doses (after 11-12 weeks; Fig. 2a). After four doses, good response was recorded for $93.8 \%(15 / 16)$ of the patients and the remission rate reached $81.3 \%$ (13/16) (Fig. 2b). In the moderate and low disease activity groups, mean DAS28ESR of $4.28 \pm 0.57 \quad(n=32)$ and $2.91 \pm 0.22$ $(n=6)$, respectively, at baseline fell below the remission threshold after one dose to $2.49 \pm 0.88 \quad(n=32)$ and $1.87 \pm 0.96(n=6)$, respectively (Fig. 2a). Hundred percent of those $(n=16)$ with moderate disease activity showed good response after four doses, with a remission rate of $81.3 \%$ (13/16) (Fig. 2c). In the low-activity group, five of the six patients went into remission after the first dose and the other one after three doses. TCZ proved to be very effective regardless of baseline disease activity. 

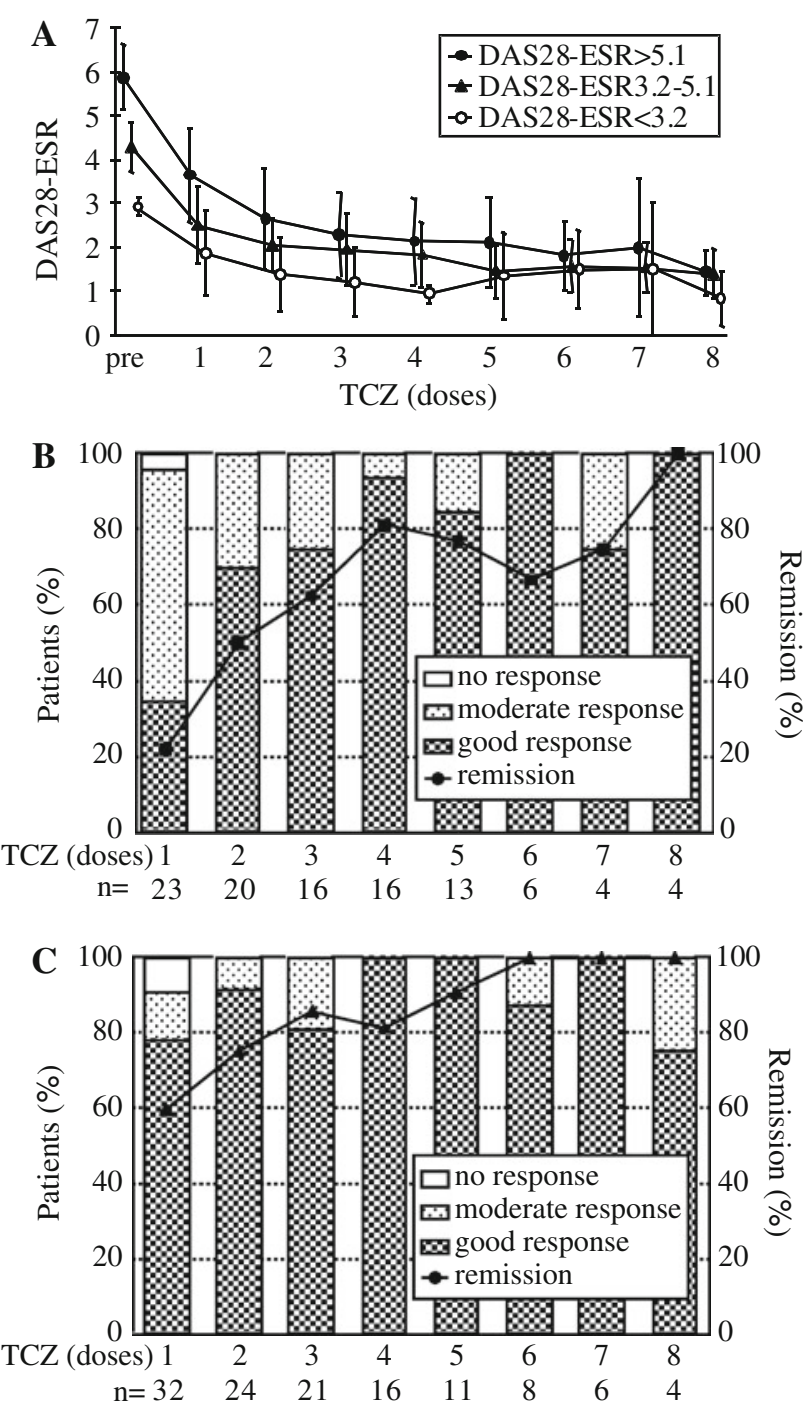

Fig. 2 a Change in DAS28ESR by disease activity at baseline. Patients were divided into three groups according to their DAS28ESR score into high disease activity (DAS28ESR $>5.1$ ), moderate disease activity $(3.2-5.1)$, and low disease activity $(2.6$ to $<3.2)$. Mean values shown. Bars indicate SD. b Change in response and remission rate in DAS28ESR $>5.1$ group. " $n$ " indicates the number of patients. (See the legend for Fig. 1.) c Change in response and remission rate in DAS28ESR 3.2-5.1 group

With age, physical capacity generally declines and resistance to infections diminishes, so that there is often more concurrent disease as well. In the joints especially, one sees an increasing frequency of pain or swelling associated with osteoarthritis. To investigate whether such factors would produce differences in the efficacy or safety of TCZ therapy, we divided the patients into an elderly group aged 65 and over $(n=24$, mean age $72.5 \pm 5.3$, mean disease duration $11.5 \pm 10.8$ years) and non-elderly group aged less than $65(n=37$, mean age $53.2 \pm 9.5$, mean disease duration $10.0 \pm 7.6$ years) in accordance with WHO classification and compared the two groups. Mean baseline
DAS28ESR was $4.93 \pm 1.13$ in the elderly and $4.63 \pm 1.15$ in the non-elderly group $(P=0.324)$ (Fig. 3a). After two doses, mean DAS28ESR fell to $2.36 \pm 1.13(n=18)$ and $2.12 \pm 0.87(n=32)$, respectively, both below the remission threshold. At the 4-dose point, good response had been demonstrated in $100 \%$ of those $(n=25)$ in the non-elderly group, with remission of $88.0 \%$ (22/25) (Fig. 3b), and in the elderly group, there had been a good response in $91.7 \%$ (11/12) with remission of $75.0 \%$ (9/12) (Fig. 3c). No significant difference between mean DAS28ESR was observed between both groups (non-elderly 1.66 versus elderly 2.21 ; $P=0.138$ ). TCZ thus provided similarly high success rates in both groups.
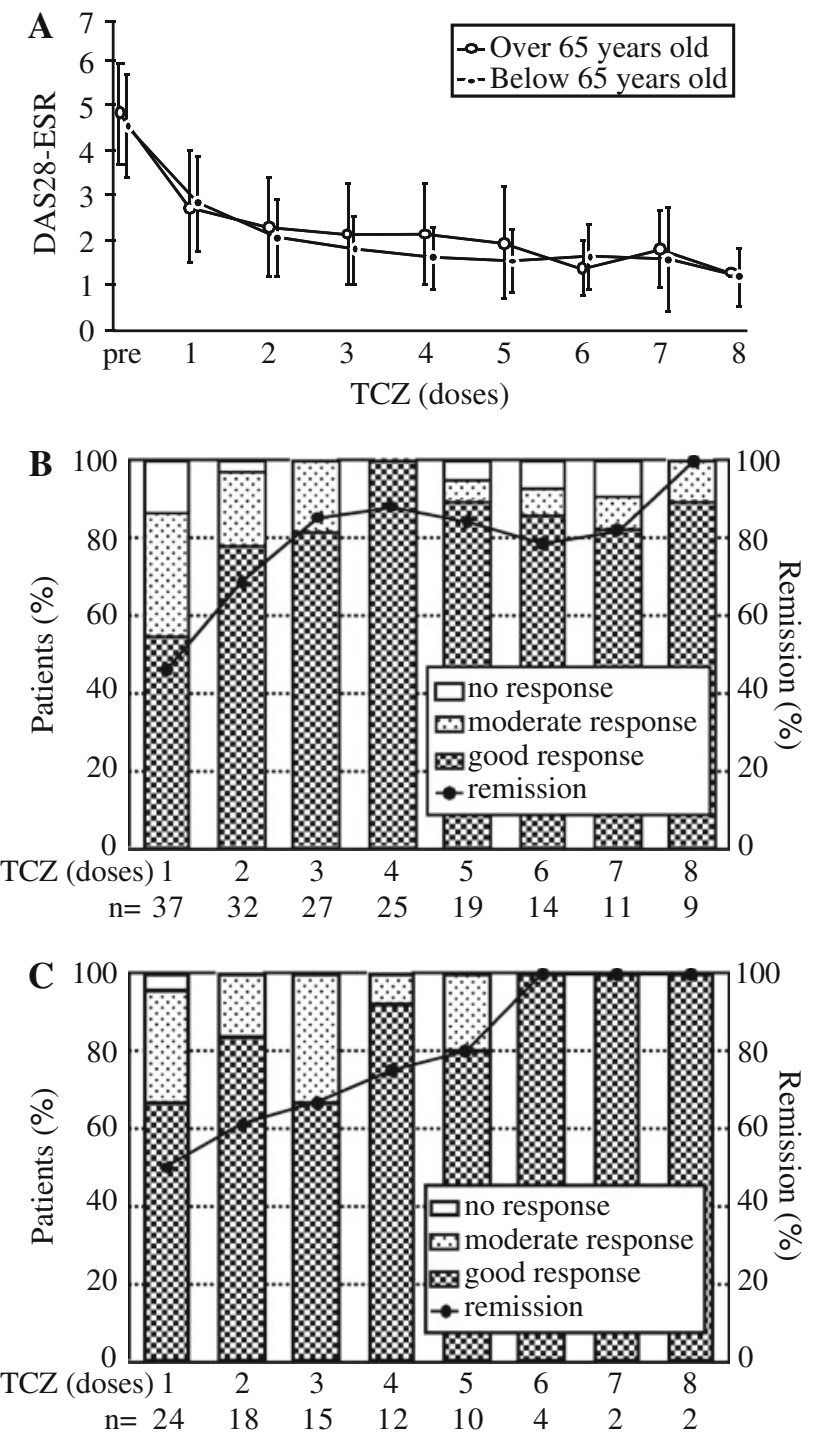

Fig. 3 a Change in DAS28ESR by age group. Mean values shown. Bars indicate SD. b Change in response and remission rate in those aged $<65$. " $n$ " indicates the number of patients. (See the legend for Fig. 1.) c Change in response and remission rate in those aged 65 and over 
Safety

A total of 27 adverse events occurred in 24 of the 61 patients and these are listed separately for the non-elderly group and elderly group in Table 2 . Taking the causal relationships discussed below, there seemed to be no difference in adverse events between the $\geq 65$ and $<65$ groups.

Anxiety disorders, palpitations, and cystitis had been seen occasionally before exposure to TCZ. Leukocytopenia reversed upon discontinuing the MTX that the patient had remained on since before treatment and giving folic acid. From the symptoms and clinical course, the nasopharyngitis and gastroenteritis seemed to be viral infections prevalent at the time. The elevated CRP and ESR appeared to be linked to inflammation from concurrent hemorrhoids. We established subsequently that a rash on the right forearm had been due to an alcohol-based disinfectant. Epigastric pain disappeared upon discontinuing the nonsteroidal anti-inflammatory drugs that the patient had been using and giving a proton pump inhibitor. We carried out upper gastrointestinal investigations and abdominal ultrasound on a patient who complained of abdominal discomfort but found nothing unusual. Although we were unable to identify the cause, it disappeared in a little while.

Table 2 Adverse events

\begin{tabular}{lll}
\hline & Age $\geq 65(n=37)$ & Age $<65(n=24)$ \\
\hline Cerebellar infarction $^{\mathrm{a}}$ & $1(2.7)$ & \\
Speech disorder $^{\mathrm{a}}$ & $1(2.7)$ & \\
Nasopharyngitis & $1(2.7)$ & \\
Anxiety disorder & $1(2.7)$ & $1(4.2)$ \\
Choking sensation & & $1(4.2)$ \\
Increased hemosputum & & $1(4.2)$ \\
Pneumonia $^{\mathrm{a}}$ & $1(2.7)$ & $1(4.2)$ \\
Palpitation & & $1(4.2)$ \\
Malignant lymphoma & & \\
Rash (right forearm) & & $1(4.2)$ \\
Epigastric pain & $1(2.7)$ & \\
Abdominal discomfort & $1(2.7)$ & \\
Gastroenteritis & $1(2.7)$ & $2(8.3)$ \\
Back pain & & $2(8.3)$ \\
Lumbar discomfort & & $1(4.2)$ \\
Ischial pain & & $1(4.2)$ \\
Cystitis & $1(2.7)$ & $2(8.3)$ \\
Leukocytopenia & & $1(4.2)$ \\
Elevated CRP & & \\
Elevated ESR & & \\
Total & $9(24.3)$ & \\
\hline
\end{tabular}

The values shown denote the number $(\%)$ of patients

a Serious adverse event
Drug-related infusion reactions were observed as eight events in five patients. The adverse events seen during infusion were transient back pain, lumbar discomfort, and ischial pain. These all appeared a few minutes into infusion and disappeared within a few minutes. There was nothing abnormal as regards body temperature, blood pressure, and pulse, nor was it the case that they occurred at each infusion. The patients were negative for IgE anti-TCZ antibodies. One patient suffered a transient mild choking sensation during the night after the first dose, but this did not recur at the time of the second and the patient was negative for $\operatorname{IgE}$ anti-TCZ antibodies as well.

Five serious adverse events occurred in total. Two patients developed pneumonia. One of these was a 60 -yearold woman with old tuberculosis and bronchiectasis as comorbidities. She showed a slight rise over her previous level of hemosputum, and bacterial pneumonia appeared subsequently. The other patient was an 80-year-old woman suspected to have pneumonia due to Chlamydia pneumoniae. Both recovered with treatment. The case of speech disorder was due to motor impairment of the tongue, but a cranial MRI scan failed to identify anything unusual. This is still being investigated as possible RA damage to the cervical vertebrae. The patient who died due to cerebellar infarction was elderly, at 82 years old, and had once suffered strokes in the past. The patient in whom malignant lymphoma became patent is considered in the Discussion. All except the two patients who suffered cerebellar infarction and malignant lymphoma are continuing to be treated with TCZ.

Laboratory findings

CRP virtually normalized in all patients after the first dose of TCZ $(1.74 \pm 1.7 \rightarrow 0.23 \pm 0.37 \mathrm{mg} / \mathrm{dl})$. ESR likewise normalized quickly after the first dose $(39.1 \pm 25.3 \rightarrow$ $8.9 \pm 9.2 \mathrm{~mm} / \mathrm{h}$ ). Both followed a normal course afterwards as well.

The mean leukocyte count was $8,400 \pm 2,990 / \mu l$ at baseline, but went down to around $6,000 / \mu \mathrm{l}$ after the first dose. There was no progressive fall as seen in myelosuppression and no significant increase in infections was recorded either (Fig. 4a). Likewise, the mean platelet count of $315,000 \pm 74,000 / \mu 1$ at baseline went to around $220,000 / \mu \mathrm{l}$ after the first dose (Fig. 4a).

The hemoglobin level rose gradually and anemia improved after treatment with TCZ began (Fig. 4b).

Figure $4 \mathrm{c}$ shows changes over time in ALT as an index of liver function. Even though abnormal values for ALT occurred, they were transient and all were of grade I based on the National Cancer Institute Common Toxicity Criteria (NCI-CTC). 

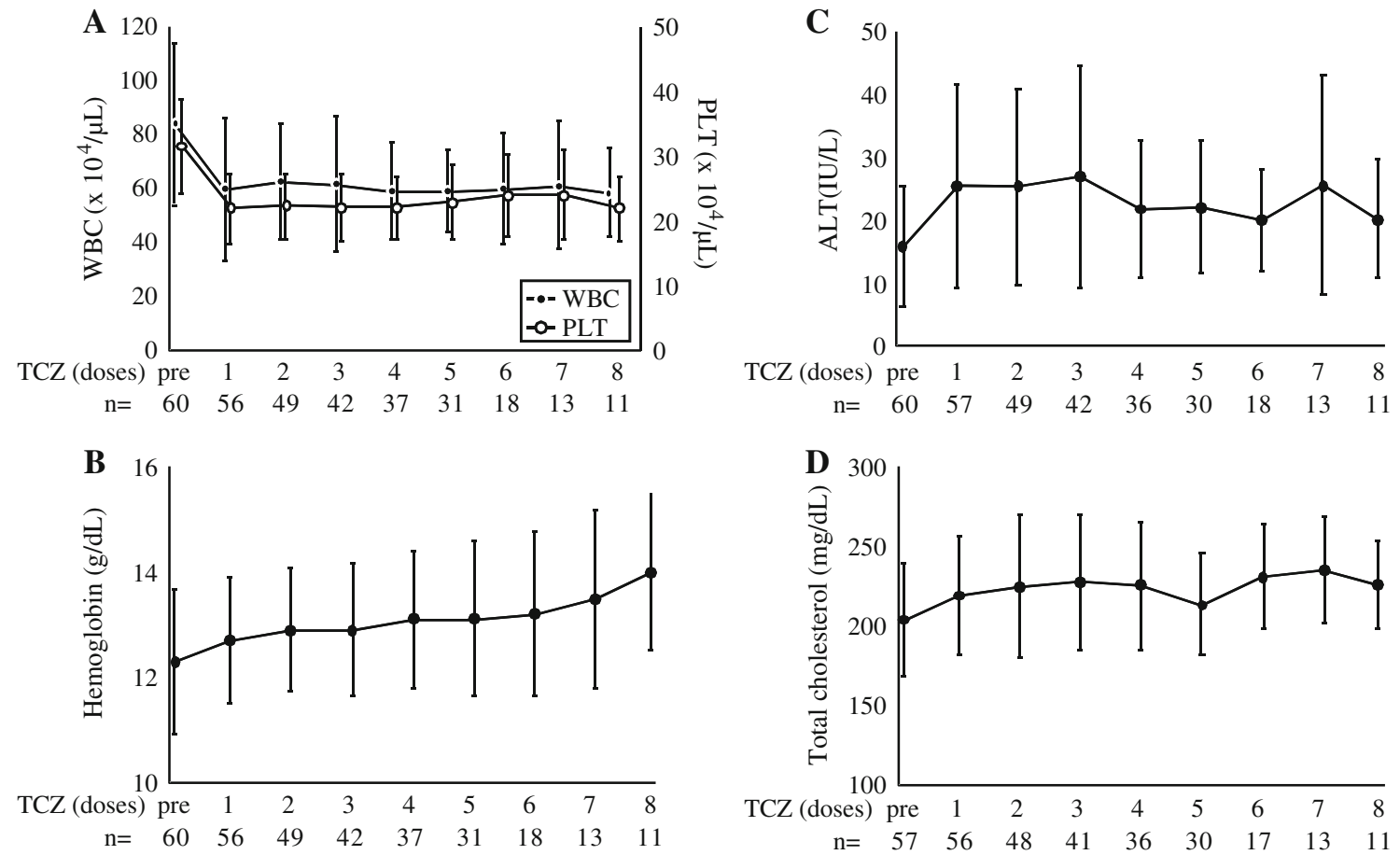

Fig. 4 Change in white blood cell counts and platelet counts (a), hemoglobin (b), alanine transaminase (c), and total cholesterol (d). Mean values shown. Bars indicate SD. " $n$ " indicates the number of patients. (See the legend for Fig. 1.)

Mean total cholesterol at baseline was $203.7 \pm 35.6 \mathrm{mg} / \mathrm{dl}$. Upon treatment with TCZ, it rose slightly and stayed around the $225 \mathrm{mg} / \mathrm{dl}$ level, but showed no continuous increase (Fig. 4d). We gave lifestyle guidance and simply monitored the clinical course without providing any drug treatment at least until dose 3 . We started five patients whose level nevertheless exceeded $280 \mathrm{mg} / \mathrm{dl}$ on treatment with a HMG-CoA reductase inhibitor.

\section{Discussion}

This article presents our therapeutic results for the use of TCZ in patients with diverse medical histories in real clinical practice. TCZ rapidly reduced disease activity in patients with RA and achieved high remission rates even in real clinical practice. A further noteworthy point was that all 61 of the patients responded to treatment. No primary or secondary failure has been seen and mean DAS28ESR has remained good (1.6-1.7) in observations until now (July 2009).

The great advantage of treatment with TCZ is that a satisfactory therapeutic response can be obtained by monotherapy [9]. As TCZ is an antibody, it has no intrinsic chemical toxicity. The frequency of infusion reactions is also small (7.0\% in the SAMURAI study) [8]. Controlling comorbidities and preventing infection are thus key issues in reducing adverse events and deriving the maximum possible therapeutic benefit from TCZ. We screened the patients for comorbidities, particularly infection, other than RA before initiating treatment with TCZ and cured or controlled these to the extent possible. This not only served to reduce adverse events, but also stopped stimulation for IL-6 production from factors other than RA. For example, periodontitis stimulates the expression of pro-inflammatory cytokines such as TNF and IL-6 [10]. Non-surgical periodontal therapy had a beneficial effect on the signs and symptoms of RA, regardless of the medications used to treat this condition [11].

Arthralgia due to infection is all too common and sometimes can elicit 'reactive arthritis' in the broad sense of the term [12]. If RA patients have reactive arthritis as a complicating factor, there is a risk of it being mistaken for treatment-refractory RA. Many types of pathogen such as the Streptococci responsible for rheumatic fever, Salmonella and Chlamydia have been reported as causative agents in reactive arthritis. All too often, however, it is difficult to identify the seat of infection. Patients in whom concomitant infection could be ruled out were thus given a 7-14 day course of $100 \mathrm{mg} /$ day doxycycline, which is even effective against Chlamydia, and we monitored whether or not their symptoms or laboratory findings improved as a result of this.

Turning to patient education, as infection control, we advised the patients about maintaining cleanliness through hand washing and gargling and avoiding contact with 
people with infections. We advised that particular attention should be paid to the cleanliness of the mouth, nostrils, urinary organs, the area around anus, and so on where pathogens can easily gain access. The risk of infection can be reduced quite substantially by keeping these areas clean. For example, ensuring good oral hygiene in nursing homes has been shown to reduce the incidence of pneumonia and mortality rates significantly [13]. In the SAMURAI study, nasopharyngitis was the most common adverse event in the both groups (the control group $32.4 \%$ (47/145), the TCZ group $35.7 \%$ (56/157)) [8]. In this report, the frequency of infections (8.2\% (5/61): 1 with nasopharyngitis, 2 with pneumonia, 1 with gastroenteritis, and 1 with cyctitis) was lower than found in the clinical trials regardless of the fact that many of our patients were elderly and that the treatment period had included the winter.

It is not clear yet whether TCZ treatment might influence the incidence of malignancies or not. The patient with malignant lymphoma discovered as a left axillary mass had undergone a PET-CT scan to assess the activity of interstitial pneumonia before being treated with TCZ. Although there were no accumulations in the lungs, accumulation was picked up in the left axillary lymph nodes. Tiny lymphadenopathy was found at the same site by CT scan. Retrospectively, it may be inferred that the accumulation seen by PET had been in the early stages of malignant lymphoma. Further investigation will be needed into whether or not TCZ has any bearing on the frequency and growth rates of malignant lymphomas.

We encountered no laboratory test abnormalities so severe that treatment with TCZ could not continue. The $\mathrm{WBC}$ and platelet counts fell following the first dose to steady levels. These matched the time courses of CRP or ESR, and we inferred that this constituted the disappearance of the additional amounts induced by stimulation from inflammatory cytokines or inflammatory chemokines, that is to say, their return to normal levels. It appeared that the rise in hemoglobin had likewise resulted from the elimination of an IL-6-linked rise in hepcidin due to the action of TCZ [14]. TCZ had no adverse effect on the blood cell system and rather reduced abnormalities brought about by RA. Slight, transient rises in ALT were seen after giving TCZ. In some patients, this improved upon discontinuing the methotrexate they had remained on since before treatment with TCZ. Some patients improved with diet therapy. Weight gain was in fact often seen, and it seemed possible that rises in ALT due to fatty liver had been involved in patients showing normalization in ALT through diet. Total cholesterol also showed a slight rise in many patients after they received TCZ, as observed similarly in the results of the clinical trials to date [6-9]. The details of the mechanism whereby TCZ produces rises in cholesterol are unclear, but in patients who improved with lifestyle guidance including diet, this was inferred to be due to the calorie excess.

During the clinical trials, we encountered several patients whose arthritis had improved following the first dose but returned after 3 weeks. This suggested that the blood level could not be maintained over the 4-week period. We thus decided to give only the second dose after an interval of 3 weeks following the first. By doing this, not one of our patients suffered repeat deterioration by the time of the second dose in symptoms that had improved following the first. A shorter dose interval at the start may be thought to be perfectly reasonable in terms of maintaining the blood levels of biologics. In short, it seems that by giving the second dose before the blood level has had a chance to fall, the time to remission can be reduced. We have not seen any rise in adverse events as a result of this.

Another article describes how bone destruction in joints that had become inflamed was demonstrated 4 months later by MRI in about half the patients [15]. It is therefore important to achieve remission within 4 months at least of the onset of RA to try and ensure that bone destruction does not set in. Based on the findings in this article, in which all patients responded to treatment and a remission rate of $83.8 \%$ was achieved after four doses, it seems that by providing treatment with TCZ from the outset, the window of opportunity for halting bone destruction in a high proportion of patients will not be lost. TCZ would seem to be promising as a first-line choice for the treatment of RA.

Open Access This article is distributed under the terms of the Creative Commons Attribution Noncommercial License which permits any noncommercial use, distribution, and reproduction in any medium, provided the original author(s) and source are credited.

\section{References}

1. Brennan FM, McInnes IB (2008) Evidence that cytokines play a role in rheumatoid arthritis. J Clin Invest 118:3537-3545

2. Alonso-Ruiz A, Pijoan JI, Ansuategui E, Urkaregi A, Calabozo M, Quintana A (2008) Tumor necrosis factor alpha drugs in rheumatoid arthritis: systematic review and metaanalysis of efficacy and safety. BMC Musculoskelet Disord 9:1-27

3. van der Heijde D, Klareskog L, Landewé R, Bruyn GA, Cantagrel A, Durez P, Herrero-Beaumont G, Molad Y, Codreanu C, Valentini G, Zahora R, Pedersen R, MacPeek D, Wajdula J, Fatenejad S (2007) Disease remission and sustained halting of radiographic progression with combination etanercept and methotrexate in patients with rheumatoid arthritis. Arthritis Rheum 56:3928-3939

4. Sato K, Tsuchiya M, Saldanha J, Koishihara Y, Ohsugi Y, Kishimoto T, Bendig MM (1993) Reshaping a human antibody to inhibit the interleukin 6-dependent tumor cell growth. Cancer Res 53:851-856

5. Ohsugi Y (2007) Recent advances in immunopathophysiology of interleukin-6: an innovative therapeutic drug, tocilizumab (recombinant humanized anti-human interleukin-6 receptor antibody), unveils the mysterious etiology of immune-mediated inflammatory diseases. Biol Pharm Bull 30:2001-2006 
6. Nishimoto N, Miyasaka N, Yamamoto K, Kawai S, Takeuchi T, Azuma J, Kishimoto T (2009) Study of active controlled tocilizumab monotherapy for rheumatoid arthritis patients with an inadequate response to methotrexate (SATORI): significant reduction in disease activity and serum vascular endothelial growth factor by IL-6 receptor inhibition therapy. Mod Rheumatol 19:12-19

7. Smolen JS, Beaulieu A, Rubbert-Roth A, Ramos-Remus C, Rovensky J, Alecock E, Woodworth T, Alten R (2008) Effect of interleukin-6 receptor inhibition with tocilizumab in patients with rheumatoid arthritis (OPTION study): a double-blind, placebocontrolled, randomised trial. Lancet 371:987-997

8. Nishimoto N, Hashimoto J, Miyasaka N, Yamamoto K, Kawai S, Takeuchi T, Murata N, van der Heijde D, Kishimoto T (2007) Study of active controlled monotherapy used for rheumatoid arthritis, an IL-6 inhibitor (SAMURAI): evidence of clinical and radiographic benefit from an $\mathrm{x}$ ray reader-blinded randomised controlled trial of tocilizumab. Ann Rheum Dis 66:1162-1167

9. Nishimoto N, Miyasaka N, Yamamoto K, Kawai S, Takeuchi T, Azuma J (2008) Long-term safety and efficacy of tocilizumab, an anti-interleukin-6 receptor monoclonal antibody, in monotherapy, in patients with rheumatoid arthritis (the STREAM study): evidence of safety and efficacy in a 5-year extension study. Ann Rheum Dis. doi:10.1136/ard.2008.092866
10. Janket SJ, Jones JA, Meurman JH, Baird AE, Van Dyke TE (2008) Oral infection, hyperglycemia, and endothelial dysfunction. Oral Surg Oral Med Oral Pathol Oral Radiol Endod 105:173-179

11. Ortiz P, Bissada NF, Palomo L, Han YW, Al-Zahrani MS, Panneerselvam A, Askari A (2009) Periodontal therapy reduces the severity of active rheumatoid arthritis in patients treated with or without tumor necrosis factor inhibitors. J Periodontol 80:535-540

12. Hannu T, Inman R, Granfors K, Leirisalo-Repo M (2006) Reactive arthritis or post-infectious arthritis? Best Pract Res Clin Rheumatol 20:419-433

13. Yoneyama T, Yoshida M, Ohrui T, Mukaiyama H, Okamoto H, Hoshiba K, Ihara S, Yanagisawa S, Ariumi S, Morita T, Mizuno Y, Ohsawa T, Akagawa Y, Hashimoto K, Sasaki H (2002) Oral care reduces pneumonia in older patients in nursing homes. J Am Geriatr Soc 50:430-433

14. Nemeth E, Rivera S, Gabayan V, Keller C, Taudorf S, Pedersen BK, Ganz T (2004) IL-6 mediates hypoferremia of inflammation by inducing the synthesis of the iron regulatory hormone hepcidin. J Clin Invest 113:1271-1276

15. McQueen FM, Stewart N, Crabbe J, Robinson E, Yeoman S, Tan PL, McLean L (1998) Magnetic resonance imaging of the wrist in early rheumatoid arthritis reveals a high prevalence of erosions at four months after symptom onset. Ann Rheum Dis 57:350-356 